EPJ Web of Conferences 66, 02094 (2014)

DOI: $10.1051 /$ epjconf/ 20146602094

(C) Owned by the authors, published by EDP Sciences, 2014

\title{
Experimental results on the Pygmy Dipole Resonance
}

\author{
Deniz Savran ${ }^{1,2, a}$ \\ ${ }^{1}$ ExtreMe Matter Institute EMMI and Research Division, GSI Helmholtzzentrum für Schwerionenforschung, \\ Planckstr. 1, 64291 Darmstadt, Germany \\ ${ }^{2}$ Frankfurt Institute for Advanced Studies FIAS, Ruth-Moufang-Str. 1, 60438 Frankfurt am Main, Germany
}

\begin{abstract}
The so-called Pygmy Dipole Resonance, an additional structure of lowlying electric dipole strength, has attracted strong interest in the last years. Different experimental approaches have been used in the last decade in order to investigate this new interesting nuclear excitation mode. In this contribution an overview on the available experimental data is given.
\end{abstract}

\section{Introduction}

In atomic nuclei nearly the complete electric dipole (E1) strength is concentrated in the well-known Isovector Electric Dipole Giant Resonance (IVGDR). This collective excitation mode can be understood as an out-of-phase oscillation of the protons and neutrons (in a macroscopic interpretation) and has been studied in the past in various experiments, see e.g. [1] and references therein.

In the last years a structure of E1 strength located at lower excitation energies has attracted considerable interest in nuclear physics. In many nuclei additional strength is experimentally found well below the IVGDR, which is mostly denoted as Pygmy Dipole Resonance (PDR) or Pygmy Dipole Strength. The strength located in the PDR is usually on the order of a (few) percent of the IVGDR, while the centroid energy is in the vicinity of the particle emission thresholds.

Even though the PDR exhausts only a rather small fraction of the corresponding energy weighted sum rule (EWSR), it is of particular interest to test details of modern microscopic models which describe the E1 response of atomic nuclei [2]. In addition it has gained interest in the last decade due to its possible connections to the neutron skin of atomic nuclei and properties of nuclear matter [3-7] as well as reaction rates in the synthesis of heavy elements [8-10]. However, the microscopic structure of the PDR, its collectivity and the robustness of the connections to other properties mentioned above are a matter of ongoing discussions.

Experimentally the situation is not conclusive regarding the systematics of the PDR and data providing insight into its structure is still rather scarce. We have recently presented the available experimental data on the PDR in our review [11] describing in detail also the experimental approaches and their limitations. In this manuscript I summarize a few of the most important aspects and refer to [11] for a detailed discussion.

\footnotetext{
ae-mail: d.savran@gsi.de
} 


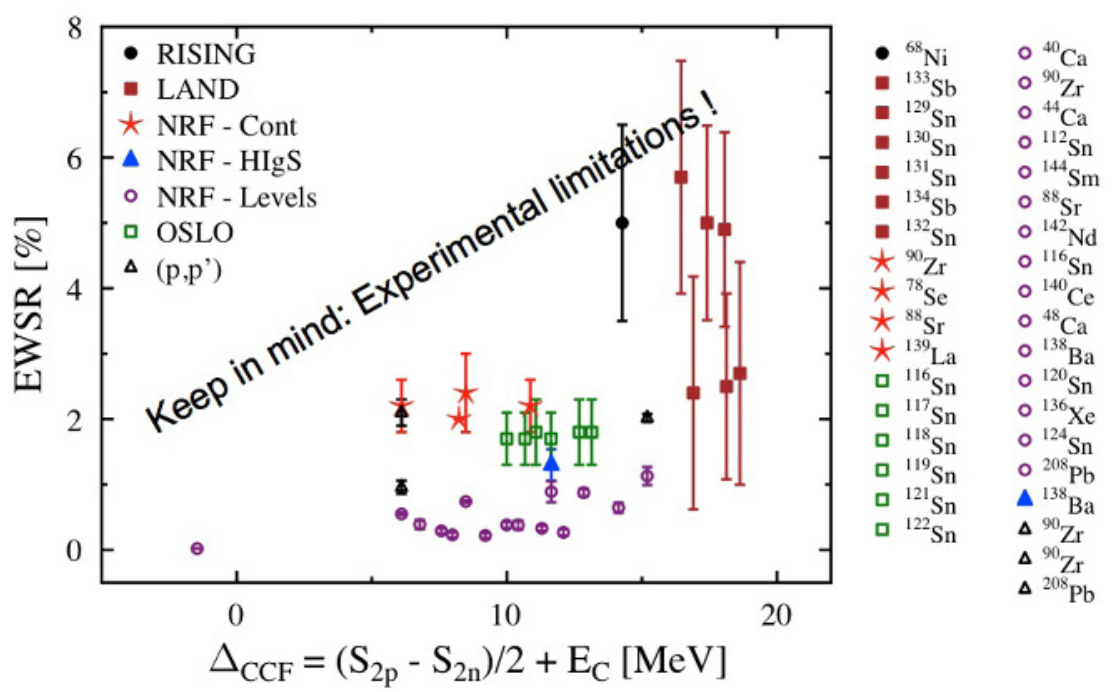

Figure 1. Reported exhaustion of the PDR in various nuclei extracted from experiments using different experimental approaches.

\section{The strength of the PDR}

Information on the (total) strength of the PDR can be obtained best in real- or virtual-photon induced reactions. On stable nuclei the nuclear resonance fluorescence (NRF) method has been most widely used to gain information on the E1 strength distribution below the neutron separation energy. For these experiments photon beams produced via bremsstrahlung [12,13] and laser Compton backscattering (LCB) [14] have been used. Different approaches in the analysis have been used taking into account either only the information of isolated peaks resulting in model-independent results but accepting a limited sensitivity (see e.g. [15]) or including the full spectra to extract the full strength but accepting the need of statistical model assumptions in the analysis and thus model dependent results (see e.g. [16]). In the energy region above the neutron threshold LCB photons have been used to measure the $(\gamma, n)$ cross section as a function of the excitation energy (see e.g. [17, 18]). Just recently a new approach has been proven to result in the extraction of the full E1 response independent of particle thresholds by the means of Coulomb excitation in the (p,p') reaction [19, 20], which however requires a separation from the M1 response via a multipole decomposition analysis.

For unstable nuclei the available approaches are much more limited. So far the E1 response in medium-heavy to heavy nuclei has been studied via Coulomb excitation in inverse kinematics in the region of ${ }^{130,132} \mathrm{Sn}[4,21]$ and in ${ }^{68} \mathrm{Ni}[22]$. Both experiments were sensitive to excitation energies above the corresponding particle thresholds. Thus, deriving a consistent systematic picture on the total strength of the PDR together with the results from stable nuclei is very difficult.

Figure 1 summarizes the reported EWSR values for a variety of nuclei of different masses (see [11] for details). However, one needs to keep in mind that the values are not derived in a consistent way, i.e. in some cases contributions of the low energy GDR tail were subtracted and different energy regions have been chosen for the integration region. This summary therefore can only provide a first idea on the strength of the PDR. In Fig. 1 the strength has been plotted vs. the parameter $\Delta_{C C F}$ which 
is defined by the difference of the two-neutron and two-proton separation energies and a correction for the Coulomb energy:

$$
\Delta_{C C F}=\left(S_{2 p}-S_{2 n}\right) / 2+E_{C}
$$

It turns out that this parameter seems to be correlated to the thickness of the neutron skin calculated in different microscopic models [11]. However, to verify this relation further investigations are necessary. Looking at Fig. 1 one does not see a clear correlation. Although for larger $\Delta_{C C F}$ also higher PDR strength is reported on average, the lack in the consistency of the extracted values does not allow to draw final conclusions. New additional complete data sets are therefore mandatory in order to derive a real systematic picture on the PDR strength as a function of $\Delta_{C C F}$ or any other parameter.

\section{Results from hadron scattering}

Photon induced reactions are the ideal choice to derive the strength of the PDR, since cross sections can be directly converted into transition strength. However, these experiments alone cannot reveal details of the underlying structure of the PDR, i.e. if the nature of a neutron skin oscillation is indeed an adequate description or if the transition densities calculated in microscopic models are reasonable. For this experiments with complementary probes are needed. It has been shown that the $\left(\alpha, \alpha^{\prime} \gamma\right)$ reaction is well suited to study low-lying bound $J^{\pi}=1^{-}$states [23]. The combination of this reaction with high-resolution $\gamma$ spectroscopy using HPGe detectors provides the feasibility of studying the PDR in $\alpha$-scattering experiments [24]. The first experiment on ${ }^{140} \mathrm{Ce}$ [25] revealed a surprising splitting of the low-lying E1 strength: While the $J^{\pi}=1^{-}$states up to about $6 \mathrm{MeV}$ are excited by $\alpha$ particles as well as photons, the higher lying $J^{\pi}=1^{-}$states are excited in the NRF reaction only. This observation is a clear indication for a change of structure in the low-lying E1 strength. The same pattern has been observed in ${ }^{94} \mathrm{Mo} \mathrm{[26],}{ }^{124} \mathrm{Sn}$ [27, 28], and ${ }^{138} \mathrm{Ba}$ [29]. Figure 2 provides an overview of the data, showing the comparison of the results of the corresponding $\left(\alpha, \alpha^{\prime} \gamma\right)$ and $\left(\gamma, \gamma^{\prime}\right)$ experiments for each nucleus. In all cases a similar splitting is observed.

The comparison to microscopic QPM and RQTBA calculations shows a qualitative agreement using the proper isoscalar (IS) E1 operator to compare to the $\left(\alpha, \alpha^{\prime} \gamma\right)$ data and the EM operator for the NRF results [27]. Based on this agreement conclusions on the nature of the E1 strength can be drawn using the calculated transition densities of the corresponding excitations. While the lower lying $J^{\pi}=1^{-}$states have transition densities showing the signature of a neutron skin oscillation, this signature is vanishing in the higher lying states, which causes the reduction of the IS E1 strength [27]. A similar reduction of the IS E1 strength compared to the EM response is also observed in other calculations $[7,30]$. The combination of experiments using complementary probes with different sensitivity to the radial dependence of the transition densities and modern microscopic calculations thus might provide for the first time an identification of the neutron skin signature of the low-lying E1 strength. However, further investigations are certainly necessary in order to verify this interpretation. Especially a proper treatment of the reaction mechanism in the comparison of the $\left(\alpha, \alpha^{\prime} \gamma\right)$ to the calculation following the concept of [31] is important.

\section{The decay properties of the PDR}

A property which can give further insight into the structure of the E1 strength, especially the coupling of complex configurations and the damping mechanism, is the decay pattern of the PDR. So far this property was not possible to directly access, since the branching transitions to individual low-lying excited states are small and a very sensitive experimental method is needed. Often the concept of 

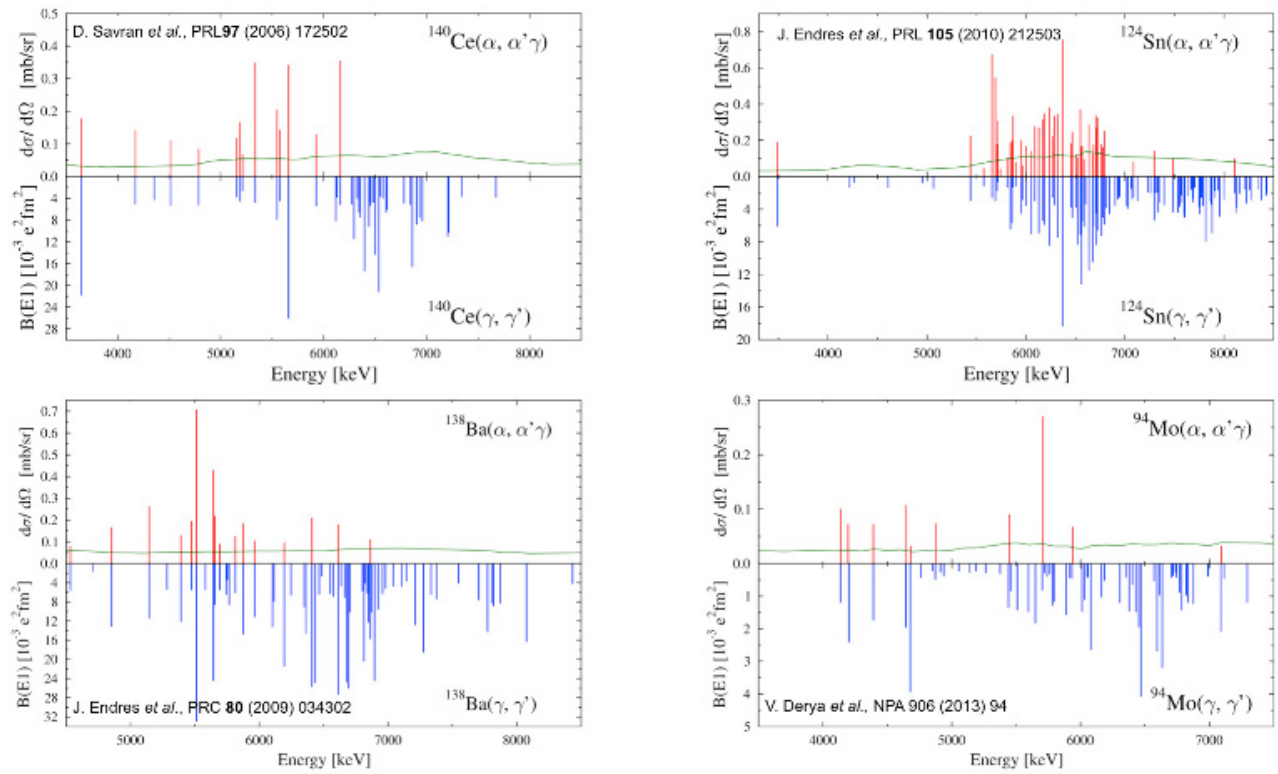

Figure 2. Results from $\left(\alpha, \alpha^{\prime} \gamma\right)$ experiments on ${ }^{94} \mathrm{Mo}[26],{ }^{124} \mathrm{Sn}[27,28],{ }^{138} \mathrm{Ba}[29]$ and ${ }^{140} \mathrm{Ce}[25,29]$.

$\gamma$-ray strength functions within the statistical model is used to describe the decay of excited states in the PDR energy region. Partly this concept is also used in the analysis of experimental data do derive information on the total strength of the PDR. An experimental determination of branching ratios and in general the decay pattern of the low-lying E1 strength is therefore of principle interest.

Standard NRF experiments are not sensitive enough to investigate the weak branching transitions to low-lying excited states, even when using a mono-energetic LCB photon beam. At the same time these NRF experiments have shown indirectly that the integrated intensity going into these so-called inelastic transitions (i.e. any transition not going directly back to the ground state) cannot be neglected [32]. The method of $\gamma-\gamma$ coincidence experiments has been shown to be very sensitive even to very weak transitions. However, so far this method has only been used in particle-induced reactions, which do not sufficiently excite the $J^{\pi}=1^{-}$states of interest. Therefore, the combination of $\gamma-\gamma$ coincidence spectroscopy and the NRF reaction using a mono-energetic photon beam from LCB offers the best possible experimental approach to access the branching pattern of the PDR.

To realize this approach the $\gamma^{3}$ collaboration has installed a new high-efficiency $\gamma$-spectroscopy setup at the High Intensity $\gamma$-ray Source $(\mathrm{HI} \gamma \mathrm{S})$ at Duke University, the new $\gamma^{3}$ setup [33]. This setup combines high-efficiency large $\mathrm{LaBr}$ Detectors with high-resolution HPGe detectors. The concept is illustrated in Fig. 3. The high efficiency of the LaBr array allows to gate on the decay of the low-lying state (green) and investigate the feeding transitions (red) with high resolution using the HPGe array. Since the photon beam is mono-energetic all transition energies are uniquely defined in a two-step decay cascade, which strongly reduces the background and, thus, provides the necessary sensitivity. In a first commissioning experiment this has been demonstrated for the case of ${ }^{32} \mathrm{~S}$ [33], where an improvement of the peak-to-background ratio of about 50 was achieved.

The first experiments to investigate the PDR in the $\mathrm{Z}=50$ and $\mathrm{N}=82$ mass regions have been performed in the end of 2012. The first results show that the sensitivity is high enough in order to 

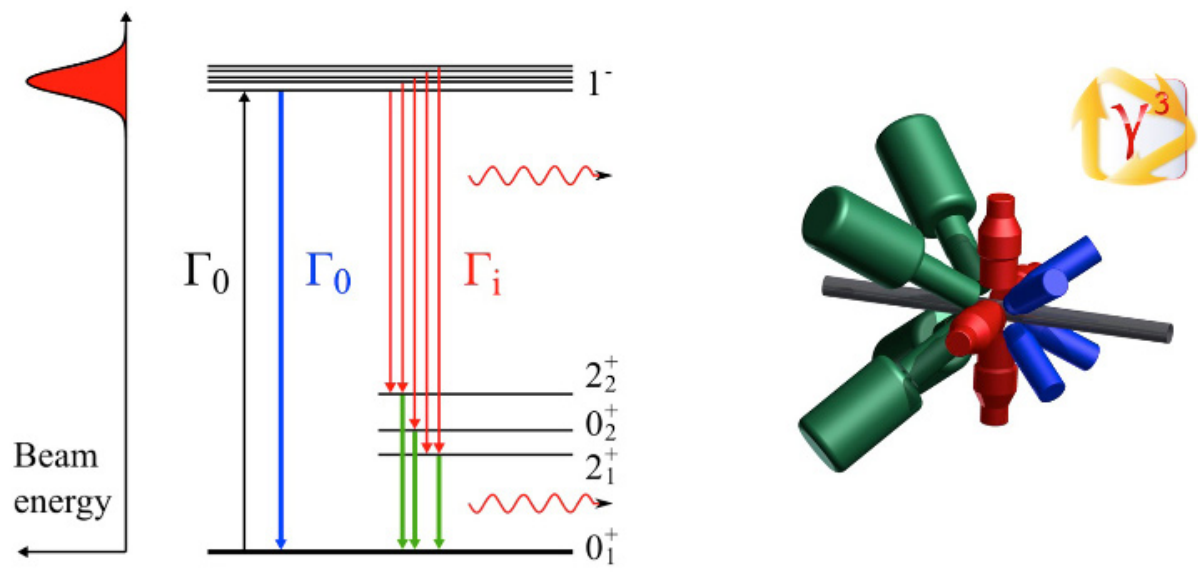

Figure 3. Concept of the $\gamma-\gamma$ coincidence technique in combination with the monoenergetic photon beam at $\mathrm{HI} \gamma \mathrm{S}$. The right part shows a schematic drawing of the new $\gamma^{3}$ setup consisting of HPGe (green) and $\mathrm{LaBr}$ detectors (red and blue) [33].

investigate directly the branching ratios into the first excited states. This new data will be confronted to microscopic calculations as well as the statistical model.

In summary, the experiments performed so far to investigate the PDR have shown, that this new excitation mode is a general phenomenon of medium-heavy to heavy nuclei. In many aspects the available experimental data is still incomplete and the open questions need to be answered by the experiments which are currently under way.

This work is supported by the Alliance Program of the Helmholtz Association (HA216/EMMI).

\section{References}

[1] M.N. Harakeh, A. van der Woude, Giant Resonances (Oxford University Press, 2001)

[2] N. Paar, D. Vretenar, E. Khan, G. Colò, Rep. Prog. Phys. 70, 691 (2007)

[3] J. Piekarewicz, Phys. Rev. C 73, 044325 (2006)

[4] A. Klimkiewicz, N. Paar, P. Adrich, M. Fallot, K. Boretzky, T. Aumann, D. Cortina-Gil, U.D. Pramanik, T.W. Elze, H. Emling et al. (LAND Collaboration), Phys. Rev. C 76, 051603 (2007)

[5] N. Tsoneva, H. Lenske, Phys. Rev. C 77, 024321 (2008)

[6] J. Piekarewicz, Phys. Rev. C 83, 034319 (2011)

[7] D. Vretenar, Y.F. Niu, N. Paar, J. Meng, Phys. Rev. C 85, 044317 (2012)

[8] S. Goriely, Phys. Lett. B 436, 10 (1998)

[9] S. Goriely, E. Khan, M. Samyn, Nucl. Phys. A739, 331 (2004)

[10] E. Litvinova, H.P. Loens, K. Langanke, G. Martinez-Pinedo, T. Rauscher, P. Ring, F.K. Thielemann, V. Tselyaev, Nucl. Phys. 823, 26 (2009) 
[11] D. Savran, T. Aumann, A. Zilges, Prog. Part. Nucl. Phys. 70, 210 (2013)

[12] R. Schwengner, R. Beyer, F. Dönau, E. Grosse, A. Hartmann, A. Junghans, S. Mallion, G. Rusev, K. Schilling, W. Schulze et al., Nucl. Instr. and Meth. A 555, 211 (2005)

[13] K. Sonnabend, D. Savran, J. Beller, M.A. Büssing, A. Constantinescu, M. Elvers, J. Endres, M. Fritzsche, J. Glorius, J. Hasper et al., Nucl. Instr. and Meth. Phys. Res. A 640, 6 (2011)

[14] H.R. Weller, M.W. Ahmed, H. Gao, W. Tornow, Y.K. Wu, M. Gai, R. Miskimen, Prog. Part. Nucl. Phys. 62, 257 (2009)

[15] D. Savran, M. Elvers, J. Endres, M. Fritzsche, B. Löher, N. Pietralla, V.Yu. Ponomarev, C. Romig, L. Schnorrenberger, K. Sonnabend et al., Phys. Rev. C 84, 024326 (2011)

[16] R. Schwengner, G. Rusev, N. Tsoneva, N. Benouaret, R. Beyer, M. Erhard, E. Grosse, A.R. Junghans, J. Klug, K. Kosev et al., Phys. Rev. C 78, 064314 (2008)

[17] H. Utsunomiya, S. Goriely, H. Akimune, H. Harada, F. Kitatani, S. Goko, H. Toyokawa, K. Yamada, T. Kondo, O. Itoh et al., Phys. Rev. C 81, 035801 (2010)

[18] H. Utsunomiya, S. Goriely, M. Kamata, H. Akimune, T. Kondo, O. Itoh, C. Iwamoto, T. Yamagata, H. Toyokawa, Y.W. Lui et al., Phys. Rev. C 84, 055805 (2011)

[19] A. Tamii, I. Poltoratska, P. von Neumann-Cosel, Y. Fujita, T. Adachi, C.A. Bertulani, J. Carter, M. Dozono, H. Fujita, K. Fujita et al., Phys. Rev. Lett. 107, 062502 (2011)

[20] C. Iwamoto, H. Utsunomiya, A. Tamii, H. Akimune, H. Nakada, T. Shima, T. Yamagata, T. Kawabata, Y. Fujita, H. Matsubara et al., Phys. Rev. Lett. 108, 262501 (2012)

[21] P. Adrich, A. Klimkiewicz, M. Fallot, K. Boretzky, T. Aumann, D. Cortina-Gil, U. Datta Pramanik, Th. W. Elze, H. Emling, H. Geissel et al. (LAND-FRS Collaboration), Phys. Rev. Lett. 95, 132501 (2005)

[22] O. Wieland, A. Bracco, F. Camera, G. Benzoni, N. Blasi, S. Brambilla, F.C.L. Crespi, S. Leoni, B. Million, R. Nicolini et al., Phys. Rev. Lett. 102, 092502 (2009)

[23] T.D. Poelhekken, S.K.B. Hesmondhalgh, H.J. Hofmann, A. van der Woude, M.N. Harakeh, Phys. Lett. B 278, 423 (1992)

[24] D. Savran, A.M. van den Berg, M.N. Harakeh, K. Ramspeck, H.J. Wörtche, A. Zilges, Nucl. Instr. and Meth. Phys. Res. A 564, 267 (2006)

[25] D. Savran, A.M. van den Berg, M.N. Harakeh, J. Hasper, A. Matic, H.J. Wörtche, A. Zilges, Phys. Rev. Lett. 97, 172502 (2006)

[26] V. Derya, J. Endres, M. Elvers, M. Harakeh, N. Pietralla, C. Romig, D. Savran, M. Scheck, F. Siebenhühner, V. Stoica et al., Nucl. Phys. 906, 94 (2013)

[27] J. Endres, E. Litvinova, D. Savran, P.A. Butler, M.N. Harakeh, S. Harissopulos, R.D. Herzberg, R. Krücken, A. Lagoyannis, N. Pietralla et al., Phys. Rev. Lett. 105, 212503 (2010)

[28] J. Endres, D. Savran, P.A. Butler, M.N. Harakeh, S. Harissopulos, R.D. Herzberg, R. Krücken, A. Lagoyannis, E. Litvinova, N. Pietralla et al., Phys. Rev. C 85, 064331 (2012)

[29] J. Endres, D. Savran, A.M. van den Berg, P. Dendooven, M. Fritzsche, M.N. Harakeh, J. Hasper, H.J. Wörtche, A. Zilges, Phys. Rev. C 80, 034302 (2009)

[30] X. Roca-Maza, G. Pozzi, M. Brenna, K. Mizuyama, G. Colò, Phys. Rev. C 85, 024601 (2012)

[31] E.G. Lanza, A. Vitturi, M.V. Andrés, F. Catara, D. Gambacurta, Phys. Rev. C 84, 064602 (2011)

[32] A.P. Tonchev, S.L. Hammond, J.H. Kelley, E. Kwan, H. Lenske, G. Rusev, W. Tornow, N. Tsoneva, Phys. Rev. Lett. 104, 072501 (2010)

[33] B. Löher, V. Derya, T. Aumann, J. Beller, N. Cooper, M. Duchene, J. Endres, E. Fiori, J. Isaak, J. Kelley et al., Nucl. Instr. and Meth. Phys. Res. A 723, 136 (2013) 